\title{
Visão da criança sobre hospitalização por meio do desenho dirigido
}

\author{
Children's visions about hospital care through drawing
}

Visión de niños/as sobre la hospitalización por el dibujo dirigido

Najara Rodrigues Dantas ${ }^{1}$, Joseph Dimas de Oliveira ${ }^{1 *}$, Simone Soares Damasceno , Vitória de Cássia Félix Rebouças ${ }^{1}$.

\section{RESUMO}

Objetivo: Conhecer a visão de crianças acerca da hospitalização por meio do desenho dirigido. Métodos: Tratou-se de um estudo qualitativo utilizando-se a técnica do desenho dirigido junto a cinco crianças. Os desenhos foram analisados a partir dos seus elementos: figuras humanas, objetos, cores e espaço físico; e à luz das ideias de Piaget. Resultados: A amostra foi composta de cinco crianças das quais uma era préescolar, três escolares e uma adolescente. As crianças desenharam 17 figuras humanas das quais 13 crianças e 4 adultos (duas mães e duas enfermeiros), seis objetos (três janelas, duas flores e uma cama), utilizaram oito cores (azul, laranja, rosa, preto, verde, cinza, marrom e amarelo) com uma média de 3,8 cores por desenho e apenas uma criança delimitou o espaço físico. Conclusão: O cuidado hospitalar é representado imageticamente englobando procedimentos, profissionais e familiares denotando que as crianças apreendem diferentes dados da realidade destacando aqueles que thes são mais representativos.

Palavras-chave: Criança hospitalizada, Enfermagem pediátrica, Desenho, Jogos e brinquedos, Criatividade.

\begin{abstract}
Objective: To know the children's visions about hospital care through drawing. Methods: It was a qualitative study using the directed drawing technique with five children. Drawings were analysed about elements on them and under Piaget's theory perspective about child development. Results: Five children one preschool child, three child and one adolescent. Children draw 17 human figure where 13 were children and 4 adults (two mothers and two nurses), six objects (three widows, two flowers and one bed), the used eight colours (blue, orange, rose, black, green, grey, brown and yellow) with a medium of 3,8 colours per drawing and only one child draw the physical space. Conclusion: We conclude that hospital care is represented broadly in images and verbally involving procedures, professionals and parents.
\end{abstract}

Keywords: Hospitalized child, Pediatric nursing, Drawing, Play and toys, Creativity.

\section{RESUMEN}

Objetivo: Conocer la mirada de niños a cerca del cuidado hospitalario a través del dibujo con dirección. Metodos: Investigación cualitativos donde se utilizó del dibujo con dirección junto a cinco niños. Resultados: Los dibujos fueron analizados a partir de los elementos presentes en ellos (figuras humanas, objetos, colores y espacio físico) y analizados a la luz de las ideas de Piaget. Resultados: La amuestra fue compuesta por cinco infantes de las cuales una era pré-escolar, tres escolares y una adolescente. Los infantes dibujaron 17 figuras humanas de las cuales 13 infantes y 4 adultos (dos madre dos enfermeras), seis objetos (tres ventanas, dos flores y una cama), utilizaron ocho colores (azul, naranja, rosa, preta, verde, gris, marrón y amarillo) con un promedio de 3,8 colores por dibujo y apenas un infante dibujó el espacio físico. Conclusión:

1 Universidade Regional do Cariri (URCA).Crato - CE. *E-mail: josephdimas@hotmail.com 
El cuidado en el hospital es representado en imagen y por las discursos de manera más amplia involucrando procedimientos, profesionales y familiares.

Palabras clave: Niño hospitalizado, Enfermería pediátrica, Dibujos, Juegos y juguetes, Creatividad.

\section{INTRODUÇÃO}

A hospitalização pode se configurar como uma experiência negativa, conflitante e desgastante para a criança devido as doenças, os procedimentos, o afastamento do cotidiano familiar e escolar e isso pode gerar alterações no comportamento como ansiedade, medo, choro e raiva (GOMES GLL, et al., 2016). No hospital, os dados fisiológicos são medidos e avaliados frequentemente, porém, ressente-se o mesmo dos dados emocionais. Nesse sentido, tem-se que os procedimentos de enfermagem podem gerar comportamentos e sentimentos negativos (CLATWORTHY S, et al., 1999; DIOGO P, et al., 2016).

Assim, cabe ao enfermeiro comunicar-se terapeuticamente com a criança e coletar dados emocionais, identificar níveis aumentados de ansiedade e intervir sobre eles ao longo da hospitalização (HOCKENBERRY MJ, WILSON D, 2018; GRAHN M, 2016). Diferentes técnicas verbais e não-verbais têm sido recomendadas para viabilizar a comunicação entre a enfermeiro e a criança nos diferentes cenários de cuidado. $O$ desenho é uma estratégia de comunicação não-verbal que pode ser conjugada à comunicação verbal. O desenho pode ser classificado como espontâneo/livre ou dirigido (HOCKENBERRY MJ, WILSON D, 2018; HARRISON LJ, 2015).

No caso do desenho livre solicita-se à criança que faça um desenho com os materiais que ela quiser (lápis de cor, pincéis hidrocor, giz de cera) e pelo tempo que ela decidir. No desenho dirigido há a solicitação para que a criança desenhe algo relacionado a algum tema e, em alguns casos, solicita-se que explique o desenho. Em técnicas mais específicas do desenho dirigido há a padronização de materiais (papel branco ou marrom, giz de cera, pincéis ou tintas guache, por exemplo) a serem disponibilizados à criança e tempo determinado (HOCKENBERRY MJ, WILSON D, 2018; HARRISON LJ, 2015).

Do ponto de vista do curso de desenvolvimento da criança, tem-se que a habilidade para se expressar por meio de atividades criativas é adquirida antes da habilidade para a leitura, a escrita e a fala mais elaboradas e, por isso, deveriam ser priorizadas quando da interação com crianças pois atuam como o canal de expressão dos sentimentos, emoções, cognição, personalidade e facilitam a comunicação entre a criança e a enfermeiro. Do ponto de vista emocional, o ato de desenhar diminui emoções como medo, raiva e ansiedade e, portanto, torna-se terapêutico. Do ponto de vista sociológico, orienta-se que pesquisas sobre crianças devam considerar a voz delas (agency) e não apenas a voz dos adultos (HARRISON LJ, 2015; HARRISON LJ, 2015; BARFIELD PA, DRIESSNACK M, 2018).

Contemporaneamente, recomenda-se a identificação e análise da visão da criança sobre os fenômenos que ela vivência. No caso da hospitalização, o interesse parte da premissa de que é um fenômeno potencialmente traumático para o qual o enfermeiro tem ferramentas para identificar e minimizar os efeitos negativos. A partir desse ponto de vista, entende-se que as construções imagéticas e verbais da criança são fontes de conhecimento e devem ser valorizados (HARRISON LJ, 2015).

Nesse sentido, o enfermeiro deve respeitar a perspectiva da criança em relação ao cuidado recebido durante a hospitalização de forma a contribuir para a construção de novas formas de produção do cuidado de enfermagem. Os pesquisadores, em particular, podem optar por uma análise de dados que valorizem os elementos visuais do desenho: cores, objetos, uso da página, figuras humanas presentes; ou a explicação verbal do desenho; ou ambos (HOCKENBERRY MJ, WILSON D, 2018; HARRISON LJ, 2015).

Apesar das três abordagens serem legítimas, observa-se que, comumente, destaca-se a adoção da segunda opção desconsiderando-se, assim, a visualidade, a criatividade e a estética do desenho; isto é, o desenho é visto apenas como meio para a coleta de dados, mas os seus elementos não são considerados úteis no momento da análise pois privilegia-se a análise das palavras da criança (HOCKENBERRY MJ, 
WILSON D, 2018; HARRISON LJ, 2015). Nesse contexto, orienta-se que o cuidado pediátrico seja centrado na criança e não no adulto; assim, os elementos não-verbais do desenho devem ser valorizados e levados em conta no momento da análise (BARIELD PA e DRIESSNACK M, 2018).

Diante disto, objetivou-se conhecer a visão de crianças acerca do cuidado hospitalar por meio do desenho dirigido.

\section{MÉTODOS}

Trata-se de um estudo descritivo, exploratório e transversal e de natureza qualitativa realizado em uma unidade de enfermaria pediátrica de um hospital de referência no interior do Estado do Ceará durante os meses de março a maio de 2013 (SANDELOWSKI M, BARROSO J, 2003; SOUSA FGM, et al., 2015).

O hospital possuía 130 leitos, atendia diferentes especialidades (clínica médica, clínica cirúrgica, unidade de terapia intensiva neonatal e adulto), tinha uma média anual de atendimentos de 64.704, dos quais 3.238 cirurgias. A unidade de internação pediátrica compreendia oito enfermarias, com 19 leitos, uma brinquedoteca e uma equipe de pediatras e enfermeiros pediatras e/ou neonatologistas, fisioterapeutas e técnicos/as de enfermagem.

A amostra foi constituída de cinco crianças internadas na Unidade Pediátrica, das quais uma pré-escolar e quatro escolares. Os critérios de inclusão foram: a) crianças em idade pré-escolar ( 3 a 6 anos) e escolar ( 6 aos 12 anos) (HARRISON LJ, 2015); b) Tempo de hospitalização de, pelo menos, 48 horas. Como critérios de exclusão: a) Crianças impossibilitadas de movimentar os membros superiores (déficit motor, déficit neurológico ou em uso de gesso ortopédico, por exemplo) que, consequentemente, inviabilizaria a participação; b) Crianças impossibilitadas de verbalizar (por trauma físico, uso de traqueostomia ou disfunção neurológica, por exemplo) o que lhes impediria de verbalizar sobre o desenho.

As crianças tinham idades entre 5 e 12 anos, sendo uma criança com cinco anos, três com 10 anos e uma com 12 anos, três do sexo masculino (Criança 2, Criança 3 e Criança 4) e duas do sexo feminino (Criança 1 e Criança 5) e que foram hospitalizadas devido a anemia falciforme, apendicite aguda, convulsão, síndrome nefrótica e lesão de pele e todas estavam acompanhadas das mães. Para a coleta de dados utilizou-se a técnica do desenho dirigido utilizando-se a seguinte questão norteadora: "Você poderia desenhar como você é cuidado aqui no hospital?". À disposição da criança havia pincéis hidrocor, lápis de cor, giz de cera, glitter, cola de papel e folhas de papel brancas. Os desenhos e as falas foram identificados por pela palavra "Criança" e enumerada pela ordem que a interação com a pesquisadora foi realizada (Criança 1, 2, 3, 5 e 5) (HOCKENBERRY MJ, WILSON D, 2018; HARRISON LJ, 2015).

Após desenharem algumas crianças comentaram sobre o que haviam desenhado voluntariamente. $O$ conteúdo verbal, no entanto, não foi o foco de análise nesse estudo já que o interesse foi o conteúdo nãoverbal. Os desenhos foram analisados a partir dos elementos presentes neles: figuras humanas, objetos, cores e espaço físico; e à luz das ideias de Piaget que divide o desenvolvimento da criança em estágios: sensório-motor, pré-operacional e operações concretas e operações formais, a partir dos quais a criança transita do aspecto individual para o social sendo essa transição intermediada pelos processos psicológicos através dos processos de assimilação (o contato com o novo) e acomodação (a aquisição de novas habilidades para reagir ao novo) (PEREIRA SR, 2011).

Respeitando-se as recomendações da Resolução 466/2012, do Conselho Nacional de Saúde, do Ministério da Saúde utilizaram-se o Termo de Assentimento (TA) junto às crianças e o Termo de Consentimento Livre e Esclarecido (TCLE) e o Termo de Imagem junto aos pais/responsáveis pela criança e a pesquisa foi submetida ao Comitê de Ética em Pesquisa (CEP) da Universidade Regional do Cariri (URCA) e aprovada sob o parecer ㄲo 103.799 (REPÚBLICA FEDERATIVA DO BRASIL, 2012).

\section{RESULTADOS}

Os elementos presentes nos desenhos das crianças são importantes pois refletem o seu mundo interno e a forma como entendem o ambiente à sua volta. Nesse sentido, os desenhos foram avaliados em relação à presença de figuras humanas, objetos, cores utilizadas e espaço físico. 
As crianças desenharam 24 elementos ao todo onde, no mínimo, uma criança desenhou três elementos (Criança 2) e, no máximo, outra criança desenhou sete elementos (Criança 5) representando uma média de 4,8 elementos por desenho - as demais crianças tiveram entre 4 a 5 elementos por desenho. Os elementos desenhados foram figuras humanas adultas e de crianças, objetos e o espaço físico.

Nos desenhos identificaram-se 17 figuras humanas (13 crianças e 4 adultos). Das 13 figuras humanas de crianças, a própria criança ou suas irmãs foram representadas no desenho enquanto que das quatro figuras humanas de adultos, duas eram a mãe e duas eram enfermeiras. Todas as cinco crianças também desenharam figuras de crianças e duas crianças desenharam figuras de adultos. As irmãs foram representadas em um desenho, conforme observa-se no Quadro 1.

Quadro 1 - Elementos dos desenhos de crianças hospitalizadas, $n=5$.

\begin{tabular}{|c|c|c|c|c|c|c|}
\hline Criança & $\begin{array}{l}\text { Doença de } \\
\text { base }\end{array}$ & Desenho & $\begin{array}{l}\text { Figuras } \\
\text { humanas }\end{array}$ & Objetos & Cores & $\begin{array}{l}\text { Espaço } \\
\text { físico }\end{array}$ \\
\hline $\begin{array}{c}\text { Criança } \\
1 \\
\text { (5 anos) }\end{array}$ & $\begin{array}{l}\text { Síndrome } \\
\text { nefrótica }\end{array}$ & & 4 crianças & 1 flor & $\begin{array}{l}\text { Azul, verde, } \\
\text { laranja, } \\
\text { rosa, cinza, } \\
\text { marrom (6) }\end{array}$ & $\begin{array}{c}\text { Não- } \\
\text { delimitado }\end{array}$ \\
\hline $\begin{array}{c}\text { Criança } \\
2 \\
\text { (10 anos) }\end{array}$ & $\begin{array}{l}\text { Convulsão } \\
\text { febril }\end{array}$ & & $\begin{array}{c}1 \text { adulto } \\
2 \text { crianças }\end{array}$ & Nenhum & $\begin{array}{l}\text { Amarelo, } \\
\text { preto e rosa } \\
\text { (3) }\end{array}$ & $\begin{array}{c}\text { Não- } \\
\text { delimitado }\end{array}$ \\
\hline $\begin{array}{c}\text { Criança } \\
3 \\
\text { (10 anos) }\end{array}$ & $\begin{array}{l}\text { Lesão de } \\
\text { pele } \\
\text { (mordedura } \\
\text { de animal } \\
\text { doméstico) }\end{array}$ & & $\begin{array}{c}1 \text { adulto } \\
2 \text { crianças }\end{array}$ & $\begin{array}{l}1 \text { flor }+ \\
1 \text { cama }\end{array}$ & $\begin{array}{l}\text { Laranja, } \\
\text { azul, rosa e } \\
\text { preto (4) }\end{array}$ & $\begin{array}{c}\text { Não- } \\
\text { delimitado }\end{array}$ \\
\hline $\begin{array}{c}\text { Criança } \\
4 \\
\text { (10 anos) }\end{array}$ & $\begin{array}{c}\text { Anemia } \\
\text { falciforme } \\
+ \\
\text { pneumonia }\end{array}$ & & 4 crianças & Nenhum & $\begin{array}{l}\text { Verde, azul } \\
\text { e laranja (3) }\end{array}$ & $\begin{array}{c}\text { Não- } \\
\text { delimitado }\end{array}$ \\
\hline $\begin{array}{c}\text { Criança } \\
5 \\
\text { (12 anos) }\end{array}$ & $\begin{array}{c}\text { Apendicite } \\
\text { aguda }\end{array}$ & & $\begin{array}{l}2 \text { adultos } \\
1 \text { criança }\end{array}$ & $\begin{array}{c}3 \\
\text { janelas }\end{array}$ & $\begin{array}{l}\text { Preto e azul } \\
\text { claro (3) }\end{array}$ & Enfermaria \\
\hline
\end{tabular}

Fonte: Dantas NR, et al., 2020. 
Em relação aos objetos desenhados tem-se janelas (3), flores (1) e cama (1). Duas crianças (Criança 2 e 4) não desenharam objetos. O máximo foi um desenho com três objetos e o mínimo foi de dois desenhos com nenhum objeto o que resulta em um média de um objeto por desenho. As cinco crianças utilizaram oito cores nos desenhos onde, no mínimo, foram utilizadas três cores (Criança 2) e, no máximo, seis cores (Criança 1) com uma média, portanto, de 3,8 cores por desenho. As cores utilizadas nos desenhos foram azuis (4 desenhos), preto, rosa e laranja (3 desenhos), verde (2 desenhos) e amarelo, cinza e marrom (1 desenho, cada). Assim, a cor mais utilizada foi azul e as menos utilizadas foram amarelos, cinza e marrom.

Em relação ao espaço físico, quatro crianças não desenharam nenhum espaço físico (Criança 1, Criança 2, 3 e 4) e uma criança desenhou a enfermaria (Criança 5). Nesse último caso, a criança utilizou a folha no sentido paisagem e desenhou a enfermaria na parte superior da folha ocupando cerca de $75 \%$ da página, a perspectiva utilizada foi a frontal (ou seja, a criança desenhou o espaço como se estivesse olhando-o de frente).

As crianças participantes desse estudo tinham idade entre cinco a 12 anos, portanto, em diferentes estágios de desenvolvimento, isto é no estágio pré-operacional (criança de 5 anos), estágio de operações concretas (as três crianças com 10 anos) e estágio operações formais (a criança de 12 anos). No primeiro caso, a característica principal é que as crianças adquirem linguagem e função simbólica além de apresentar o pensamento egocêntrico, lúdico e mágico. A representação de crianças que retrata o pensamento centrado em si mesma e o fato de ter desenhado uma flor e utilizado seis cores cumprem a função simbólica.

No caso das três crianças que estão no estágio de operações concretas espera-se que apresentem pensamento mais lógico, coerente, organizado, onde a simbologia mantém relação direta com a realidade concreta e, por isso, os conceitos surgem das experiências prévias. No nosso estudo, elas desenharam duas figuras humanas de adultos, nove figuras humanas de crianças, seis objetos e utilizaram, em média, três cores. O que foi representado de fato mantém relação com a realidade próxima das crianças (adultos, crianças, cama e janelas, uso de cores primárias como preto, azul e verde) ao mesmo tempo em que há algo do simbólico presente (flores).

No caso da criança de 12 anos e que está, portanto, no estágio formal espera-se esquemas conceituais abstratos onde as ideias desempenham papel central na forma como o indivíduo apreende a realidade. Assim, ideias abstratas como amor, carinho, religião, democracia, por exemplo, passam a ter sentido e irão embasar os comportamentos, sentimentos e atitudes ao longo da vida onde cada indivíduo buscará se encontrar em si mesmo.

No nosso estudo, a criança nesse estágio desenhou duas figuras humanas adultas, uma figura humana criança, três janelas e utilizou três cores. $O$ fato de ter desenhado mais figuras humanas adultas assim como ter desenhado janelas reflete elementos do mundo adulto, do qual se deseja ter independência. Sobretudo o ato de desenhar janelas que, como se viu, simbolizam o desejo de deslocar-se de um ambiente para outro.

\section{DISCUSSÃO}

O ato de desenhar é importante porque é centrado na criança, tem efeito terapêutico, ajuda na identificação com o ambiente externo e tem-se mostrado útil para acessar conteúdos emocionais e sensíveis, como a hospitalização (HARRISON LJ, 2015; MAYABA NN, WOOD L, 2015; POPE N, et al., 2017). O desenho, enquanto uma técnica projetiva, permite identificar os elementos que produzem significado para a criança. Comumente, as crianças hospitalizadas desenham figuras humanas e objetos que cumprem esse papel de significado (ALBA-LEONEL A, et al., 2018; ÇALBA-LEONEL A, et al., 2018).

As figuras humanas geralmente presentes nos desenhos de crianças hospitalizadas são os profissionais, os pais e as próprias crianças, enquanto que os objetos podem incluir objetos de casa ou materiais hospitalares (ALBA-LEONEL A, et al., 2018; ÇALBA-LEONEL A, et al., 2018). No presente estudo, os desenhos das crianças representaram figuras de familiares, profissionais de saúde e mobiliários hospitalares refletindo, assim, as pessoas e objetos significativos para eles/as. 
A presença de figuras humanas familiares reflete senso de segurança da criança em relação aos adultos e/ou a si mesma (CLATWORTHY S, et al., 1999). Assim, no nosso estudo as figuras humanas femininas da família e das profissionais representam a fonte de segurança emocional dos participantes. Particularmente, a representação da mãe simboliza também segurança física e emocional - além do aspecto cultural de que é a mãe que permanece a maior parte do tempo com a criança em casa e também durante a hospitalização.

As figuras humanas representando os enfermeiros foram desenhadas na enfermaria, ao lado das crianças e usando uniformes coloridos. Esse dado corrobora outros estudos onde o enfermeiro, comumente, foi representado sorrindo, com uniformes coloridos, realizando procedimentos, divertindo as crianças, segurando seringas e/ou outros materiais hospitalares. Crianças com doenças crônicas tendem a desenhar enfermeiros e suas tarefas mais do que as demais crianças (ALBA-LEONEL A, et al., 2018; ÇALBA-LEONEL A, et al., 2018; PELANDER T, et al., 2007; COMPARCINI D, et al., 2018).

Os enfermeiros, por sua vez, devem compreender e propiciar um bom encontro com as crianças, adaptando formas de comunicação e focar na família para melhorar a qualidade do cuidado de enfermagem (GRAHN M, 2016). Entre crianças em pré-operatório, atividades de leitura de histórias em livros infantis também ajudou a diminuir o nível de ansiedade (SEKHAVATPOUR Z, et al., 2019). As crianças conseguem identificar os atributos e características que distinguem os enfermeiros das/os demais profissionais e, assim, há reconhecimento do fazer do enfermeiro nos desenhos da criança contribuindo para o fortalecimento simbólico da sua função social (KHAJEH M, et al., 2017).

Comumente, as crianças tendem a desenhar elas próprias, os pais, os enfermeiros e os médicos e reproduzem situações em que as crianças estão falando, brincando ou andando, os pais estão dispostos ao seu lado e os/as profissionais estão realizando procedimentos (ÇALBA-LEONEL A, et al., 2018; PELANDER $\mathrm{T}$, et al., 2007). Interessante notar que a autonomia esperada para a criança se pôde observar também no ato de desenhar mais outras crianças do que adultos já que nessa fase a opinião dos adultos externos passa a ter maior importância do que a dos adultos de referência (REPÚBLICA FEDERATIVA DO BRASIL, 2005). Com isso, pode-se inferir que as crianças deste estudo, se reconhecem no espaço hospitalar e representam esse senso de reconhecimento/pertencimento no desenho.

Em relação aos objetos tem-se que o fato de estarem presentes no desenho também denota a fonte de segurança para a criança. As janelas significam que a criança projeta-se para o ambiente externo já que as janelas dão a visão do mundo exterior, trazem luz natural que é mais informativa que a luz artificial e, por isso, tem efeito terapêutico (CLATWORTHY S, et al., 1999; PELANDER T, et al., 2007). No Brasil, flores não são permitidas nas enfermarias devido o risco de infecção e, com isso, a presença delas nos desenhos reflete, provavelmente, elementos do mundo simbólico da criança podendo ser um elemento puramente decorativo e/ou ideias ou desejos de tranquilidade, paz e relaxamento (REPÚBLICA FEDERATIVA DO BRASIL, 2005).

A cama faz parte do mobiliário dos hospitais e, necessariamente, pode não trazer nenhum aspecto emocional negativo. A atenção deve ser dada, entretanto, à relação do tamanho da cama ao tamanho da criança pois deve haver uma proporção entre ambos já que crianças ansiosas tendem a desenhar-se deitadas e imóveis e/ou em camas muito grandes (CLATWORTHY S, et al., 1999). No presente estudo, havia duas crianças (Criança 4 e 5) com doenças crônicas (anemia falciforme e síndrome nefrótica) que, geralmente, vivenciam mais hospitalizações, realizam mais procedimentos e tem maior contato com profissionais de saúde do que outras crianças e isto pode levar a níveis maiores de ansiedade.

Sobre a utilização de cores sabe-se que há relação entre o estado emocional e a quantidade de cores que a criança decide usar. Desenhos coloridos expressam emoções positivas e desenhos com poucas cores expressam estados emocionais negativos. As cores quentes como vermelho, laranja e amarelo expressam satisfação, vitalidade e movimento, já as cores verde, azul e roxo relacionam-se com calma e as cores marrom e preta apontam níveis aumentados de ansiedade (ÇALBA-LEONEL A, et al., 2018). O roxo, no entanto, pode ser lido como aumento de ansiedade em algumas técnicas de análise (CLATWORTHY S, et al., 1999; BARBOSA AJG, et al., 2017). 
Em um estudo que comparou a ansiedade entre crianças no dia da cirurgia e crianças na escola identificou que a ansiedade foi maior no primeiro grupo e que variáveis relativas às cores (número de cores utilizadas e qualidade do traço) diferiram entre os grupos (WENNSTROM B, et al., 2018). No nosso estudo, as cores mais utilizadas (azul, laranja e verde) relacionam-se com estados emocionais positivos, apesar do preto também ter sido utilizado o que refletiu certa ambiguidade ou instabilidade emocional.

A delimitação do espaço físico pode ou não acontecer e as crianças podem desenhar o hospital como um todo, a unidade de internação, a enfermaria ou a cama (2). Outros estudos apontam que as crianças tendem a desenhar o prédio do hospital visto de fora. Crianças com altos níveis de ansiedade tendem, porém, a não desenhar o espaço físico $(2,15)$. Sobre a posição da folha, em paisagem ou retrato, orienta-se que a folha seja disponibilizada à criança em quaisquer das posições tendo a criança a liberdade de mudá-la para realizar o desenho como preferir (CLATWORTHY S, et al., 1999; HARRISON LJ, 2018; PELANDER T, et al., 2007).

Um estudo que comparou a ansiedade de crianças no dia da cirurgia com crianças na escola não identificou diferença estatisticamente significante nos desenhos dos dois grupos, porém, as crianças na escola desenharam mais o ambiente do que o outro grupo de crianças (SEKHAVATPOUR Z, et al., 2019). Outros estudos semelhantes identificaram que as crianças no hospital apresentaram níveis mais elevados de ansiedade denotando que o hospital, de fato, atua como ambiente estressor para as crianças (REPÚBLICA FEDERATIVA DO BRASIL, 2005; WENNSTROM B, et al., 2018).

A expressão através do desenho reflete a organização interna da criança e a forma como consegue perceber o mundo ao redor. Assim, à medida que uma criança cresce passa a adquirir a habilidade de desenhar de forma a refletir mais logicamente a realidade. No nosso estudo, duas crianças pré-escolares (Criança 1 e 2) encontram-se no estágio pré-esquemático de desenvolvimento ( 4 a 7 anos) no qual conseguem desenhar de forma mais elaborada do que os garatujas característicos/as da fase de desenvolvimento anterior entre 2 a 4 anos (HOCKENBERRY MJ, WILSON D, 2018; PEREIRA SR, 2011).

A Criança 3 e a Criança 5, por sua vez, estão no estágio esquemático (7 a 9 anos), enquanto a Criança 5 tem 12 anos e está no estágio de operações formais no qual a criança apresenta noções mais elaboradas da realidade e os desenhos tornam-se cada vez mais próximos do real. No estágio esquemático, a criança já consegue organizar no papel mais informações daquilo que é importante no seu cotidiano, ou seja, ela retira do seu repertório vivencial situações, pessoas e atividades e os exprimem por meio do desenho e da fala (4, 9). Dessa forma, entende-se o fato de as crianças terem desenhado a si mesmas, às mães e aos profissionais no espaço hospitalar (HOCKENBERRY MJ, WILSON D, 2018; PEREIRA SR, 2017 SANTOS PM, et al., 2016; WEGNER W, et al., 2017).

A percepção da criança acerca do mundo externo inclui a capacidade de identificar objetos, o que pode ser observado em crianças a partir de 4 meses de idade. A partir de um ano, a criança já é capaz de exteriorizar suas emoções e sentimentos através de desenhos mesmo com a ausência de forma, contornos e representação definidos. Nessa fase, os desenhos são elementares, assim como a verbalização (PEREIRA SR, 2017).

\section{CONCLUSÃO}

A visão das crianças acerca da hospitalização por meio do desenho aponta para um momento de valorização de outras crianças, uso de cores e objetos que denotaram sentimentos ambivalentes e pouca representação do espaço físico. O cuidado hospitalar é representado imageticamente englobando procedimentos, profissionais e familiares. Dentre as limitações do estudo tem-se o fato de ter sido realizado apenas um desenho com cada criança, o tamanho pequeno da amostra e, por fim, o fato dos desenhos terem sido criados apenas na enfermaria. Sugere-se, assim, que novos estudos sejam realizados ao longo da hospitalização de forma a compreendê-la como um processo sob a expressão do desenho.

\section{AGRADECIMENTOS E FINANCIAMENTO}

Fundação Cearense de Apoio ao Desenvolvimento Científico e Tecnológico (FUNCAP) pelo apoio financeiro para a realização da pesquisa. 


\section{REFERÊNCIAS}

1. ALBA-LEONEL $A$, et al. Representación e identidad de la práctica de enfermería en el dibujo. Revista de enfermería del Instituto Mexicano del Seguro Social, 2018; 26(3):186-94.

2. BARFIELD PA, DRIESSNACK M. Children with ADHD draw-and-tell about what makes their life really good. Journal for specialists in pediatric nursing, 2018; 23(2).

3. BARBOSA AJG, et al. Evidências Preliminares de Validade e Fidedignidade da Versão Brasileira do Child Drawing Hospital. Avaliação Psicológica, 2017; 16(3): 338-347.

4. CLATWORTHY S, et al. Child Drawing: Hospital Manual. Journal of Pediatric Nursing, 1999; 14(1):10-19.

5. ÇALBA-LEONEL A, et al. Investigating Children's Perception of Nurses Through Their Drawings. Clinical nursing research, 2018;17(8):1-18.

6. COMPARCINI D, et al. Children's Perceptions About the Quality of Pediatric Nursing Care: A Large Multicenter CrossSectional Study. Journal of Nursing Scholarship, 2018;50(3):287-95.

7. DIOGO P, et al. Os Medos das Crianças em Contexto de Urgência Pediátrica: Enfermeiro Enquanto Gestor Emocional. Pensar Enfermagem, 2016; 20(2):26-47.

8. GRAHN M, et al. Interactions Between Children and Pediatric Nurses at the Emergency Department: A Swedish Interview Study. Journal of Pediatric Nursing, 2016;31(1):284-92.

9. GOMES GLL, et al. Ansiedade da hospitalização em crianças: análise conceitual. Revista Brasileira de Enfermagem, 2016; 69(5):940-5.

10. HARRISON LJ. Using children's drawings as a source of data in research. Em: Harrison LJ. Handbook of research methods in early childhood education: research methodologies. $1^{\text {a }}$ ed. Charlotte: IAP, 2015. p. 433-72.

11. HOCKENBERRY MJ, WILSON D. Wong: Fundamentos de Enfermagem Pediátrica. 10 ed. Rio de Janeiro: Elsevier, 2018. 1072p.

12. KHAJEH M, et al. Family centered care of hospitalized children: A hybrid concept analysis in Iran. Health promotion perspectives, 2017; 7(4):210-5.

13. MAYABA NN, WOOD L. Using Drawings and Collages as Data Generation Methods With Children: Definitely Not Child's Play. International Journal of Qualitative Methods, 2015; 11(1):1-10.

14. PELANDER T, et al. Children in the hospital: elements of quality in drawings. Journal of Pediatric Nursing, 2007; 22(4):333-41.

15. PEREIRA SR. Educação e preparo do paciente e da família para a Terapia intravenosa. Em: HARADA MJCS, PEDREIRA MLG. Terapia intravenosa e infuses. São Caetano do Sul (SP): Yendis Editora; 2011. p. 121-36.

16. POPE N, et al. Using 'draw, write and tell' to understand children's health-related experiences. Nursing Research, 2018; 26(2):42-45.

17. REPÚBLICA FEDERATIVA DO BRASIL. Ministério da Saúde. Agência Nacional de Vigilância Sanitária. Pediatria: prevenção e controle de infecção hospitalar. Brasília: Ministério da Saúde, 2005.

18. REPÚBLICA FEDERATIVA DO BRASIL. Ministério da Saúde. Conselho Nacional de Saúde. Resolução 466/2012. Dispõe sobre normas de pesquisa com seres humanos. Diário Oficial da União, 12 de dezembro de 2012.

19. SANDELOWSKI M, BARROSO J. Classifying the Findings in Qualitative Studies. Qualitative health research, 2003; 13(7):905-23.

20. SANTOS PM, et al. Nursing care through the perception of hospitalized children. Revista Brasileira de Enfermagem, 2016; 69(4):603-9.

21. SEKHAVATPOUR $Z$, et al. The effect of storytelling on anxiety and behavioral disorders in children undergoing surgery: a randomized controlled trial. Pediatric Health, Medicine and Therapeutics, 2019;10(1):61-8.

22. SOUSA FGM, et al. Contornos conceituais e estruturais da pesquisa qualitativa. Em: Lacerda MR, Costenaro RGS. Metodologias da pesquisa para a enfermagem e saúde. Porto Alegre: Moriá; 2015. p. 99-122.

23. WENNSTROM B, et al. Evaluation of the Swedish version of the Child Drawind: Hospital Manual. Journal of Advanced Nursing, 2018; 67(5):1118-28.

24. WEGNER W, et al. Segurança do paciente no cuidado à criança hospitalizada: evidências para enfermagem pediátrica. Revista Gaúcha de Enfermagem, 2017; 38(1):1-9. 